Revista Brasileira de Fisiologia do Exercício 2018;17(3):156-64

\title{
ARTIGO ORIGINAL \\ Efeitos dos recursos da fisioterapia dermatofuncional sobre a perda de peso e sobre os marcadores de risco cardiovascular em pacientes obesos \\ Effects of resources of dermatofunctional physiotherapy on weight loss and markers of cardiovascular risk in obese patients
}

Carla Alimuse Beserra de Araújo, Ft.. ${ }^{*}$ Luciana Feitosa Holanda Queiroz, Ft. ${ }^{*}$, Adriane Sampaio Cavalcante $^{* *}$, Renata Bessa Pontes, D.Sc. ${ }^{* *}$

*Graduada pelo Centro Universitário Christus (Unichristus), Fortaleza/CE, ${ }^{* *}$ Discente do curso de Fisioterapia do Centro Universitário Christus (Unichristus) Fortaleza/CE, ${ }^{* * *}$ Orientadora do curso de Fisioterapia do Centro Universitário Christus (Unichristus) Fortaleza/CE

Recebido em 7 de maio de 2018; aceito em 18 de setembro de 2018.

Endereço de correspondência: Renata Bessa Pontes, Rua Vereador Paulo Mamede, 130, Fortaleza CE, E-mail: renatabessa@ufc.br; Carla Alimuse Beserra de Araújo: carlaalimuseba@hotmail.com; Luciana Feitosa Holanda Queiroz: luhqueiroz@hotmail.com; Adriane Sampaio Cavalcante: adrane.samp@hotmail.com;

\begin{abstract}
Resumo
Introdução: A obesidade está intimamente relacionada ao aumento da morbimortalidade, sendo considerada uma epidemia global. Objetivos: Esta pesquisa teve como objetivo analisar os efeitos dos recursos da fisioterapia dermatofuncional sobre a perda de peso e sobre os marcadores de risco cardiovasculares. Métodos: Foi realizada uma pesquisa comparativa, intervencionista, prospectiva de caráter quantitativo no período de agosto de 2017 a março de 2018. A população do estudo foi constituída por 19 pacientes com sobrepeso ou obesidade divididos em 2 grupos: o grupo um que recebeu acompanhamento endocrinológico e fisioterapia dermatofuncional, com os recursos de endermologia, corrente russa, massagem modeladora e ergometria e o grupo controle, sendo acompanhados somente pelo endocrinologista e avaliados antes e após três meses. Resultados: Foi constatado que o grupo submetido à fisioterapia mostrou redução de medidas de $15,4 \mathrm{~cm}$ dos dados antropométricos e de $24,4 \mathrm{~mm}$ de adipometria com significância estatística $(p<0,01)$ com redução do índice de massa corpórea e da relação cintura/quadril ao ser comparado com o grupo controle. Conclusão: Foi constatado que o grupo com tratamento endocrinológico associado às técnicas dermatofuncionais obteve melhores resultados que somente com 0 acompanhamento endocrinológico, sugerindo a importância da fisioterapia dermatofuncional sobre a perda de peso e sobre os marcadores de risco cardiovasculares.
\end{abstract}

Palavras-chave: fisioterapia, doenças cardiovasculares, obesidade.

\begin{abstract}
Introduction: Obesity is closely related to increased morbidity and mortality, being considered a global epidemic. Objectives: This study aimed to analyze the effects of dermatofunctional physical therapy resources on weight loss and on cardiovascular risk markers. Methods: A comparative, interventional, prospective, quantitative study was conducted between August 2017 and March 2018. The study population consisted of 19 overweight or obese patients divided into two groups: the one group that received endocrinological follow-up and dermatofunctional physical therapy, with the resources of endermology, Russian current, modeling massage and ergometry and the control group, being followed only by the endocrinologist and evaluated before and after three months. Results: We observed that the group submitted to physical therapy showed a reduction of $15.4 \mathrm{~cm}$ in anthropometric data and $24.4 \mathrm{~mm}$ in adipometry with statistical significance $(p<0.01)$, with reduction of body mass index and waist / hip ratio when compared to the control group. Conclusion: It was verified that the endocrinological treatment associated with the dermatofunctional techniques obtained better results than only with the endocrinological accompaniment suggesting the importance of dermatofunctional physical therapy on the weight loss and on the cardiovascular risk markers. Key-words: physical therapy specialty, cardiovascular diseases, obesity.
\end{abstract}


A prevalência da obesidade representa uma epidemia global, presente tanto em países desenvolvidos como naqueles em desenvolvimento. O sobrepeso e a obesidade estão intimamente relacionados ao aumento de morbidade e mortalidade. Mesmo com o controle dos fatores de risco, como o diabetes melitus, dislipidemias, síndrome metabólica e a hipertensão arterial, o risco de eventos cardiovasculares permanece elevado. Assim, a obesidade é considerada um fator de risco cardiovascular independente [1-3].

A obesidade e o aumento da circunferência abdominal sobrecarregam o coração, aumentando o risco de doenças cardíacas e acidente vascular encefálico. Essa sobrecarga cardíaca pode ser minimizada com mudanças no estilo de vida, técnicas cognitivocomportamentais, farmacoterapia, fisioterapia dermatofuncional e cirurgia bariátrica [4-6].

A fisioterapia dermatofuncional auxilia na redução de medidas em pacientes obesos, no pré e pós-operatório, utilizando técnicas e recursos que trazem benefícios à saúde do paciente.

O índice de massa corporal (IMC) é um dos instrumentos mais utilizados para avaliar o excesso de peso e a obesidade, porém não reflete adequadamente a distribuição de gordura corporal, o que tem sido fortemente correlacionado com risco cardiovascular. Já a gordura abdominal é um dos fatores de risco em potencial para doença cardíaca, independente da adiposidade corporal total [7,8]. Existem diversos métodos para avaliar a distribuição de gordura entre eles a relação da medida da circunferência abdominal e do quadril se destaca em virtude da sua fácil execução, assim como da sua boa correlação com precisão na medida da gordura abdominal [9].

Este estudo teve como objetivos analisar os efeitos dos recursos terapêuticos da fisioterapia dermatofuncional sobre a perda de peso e sobre os marcadores de risco cardiovascular em pacientes obesos, por meio de recursos fisioterápicos dermatofuncionais e analisar a resposta dos pacientes obesos ao tratamento fisioterápico.

\section{Material e métodos}

Tratou-se de uma pesquisa de caráter quantitativo, comparativa, intervencionista e prospectiva. O estudo foi realizado no ambulatório de endocrinologia e no laboratório de fisioterapia dermatofuncional do Centro Universitário Christus, no período de agosto de 2017 a março de 2018.

Foram incluídos vinte pacientes de ambos os sexos na faixa etária de 20 a 40 anos com sobrepeso ou obesidade que aceitaram se submeter à entrevista inicial de avaliação e reavaliação. Do total foi realizada uma amostragem aleatória. Dez pacientes foram encaminhados ao ambulatório de fisioterapia dermatofuncional com o objetivo de iniciar tratamento com recursos fisioterápicos dermatofuncionais (programa ergométrico e estético) formando o grupo um (g1) durante três meses. Enquanto os outros dez indivíduos que constituíram o grupo controle (g2) foram avaliados e reavaliados ao final da pesquisa para acompanhamento, sem, entretanto, serem realizadas condutas fisioterápicas, mas com 0 tratamento endocrinológico (figura 1). 


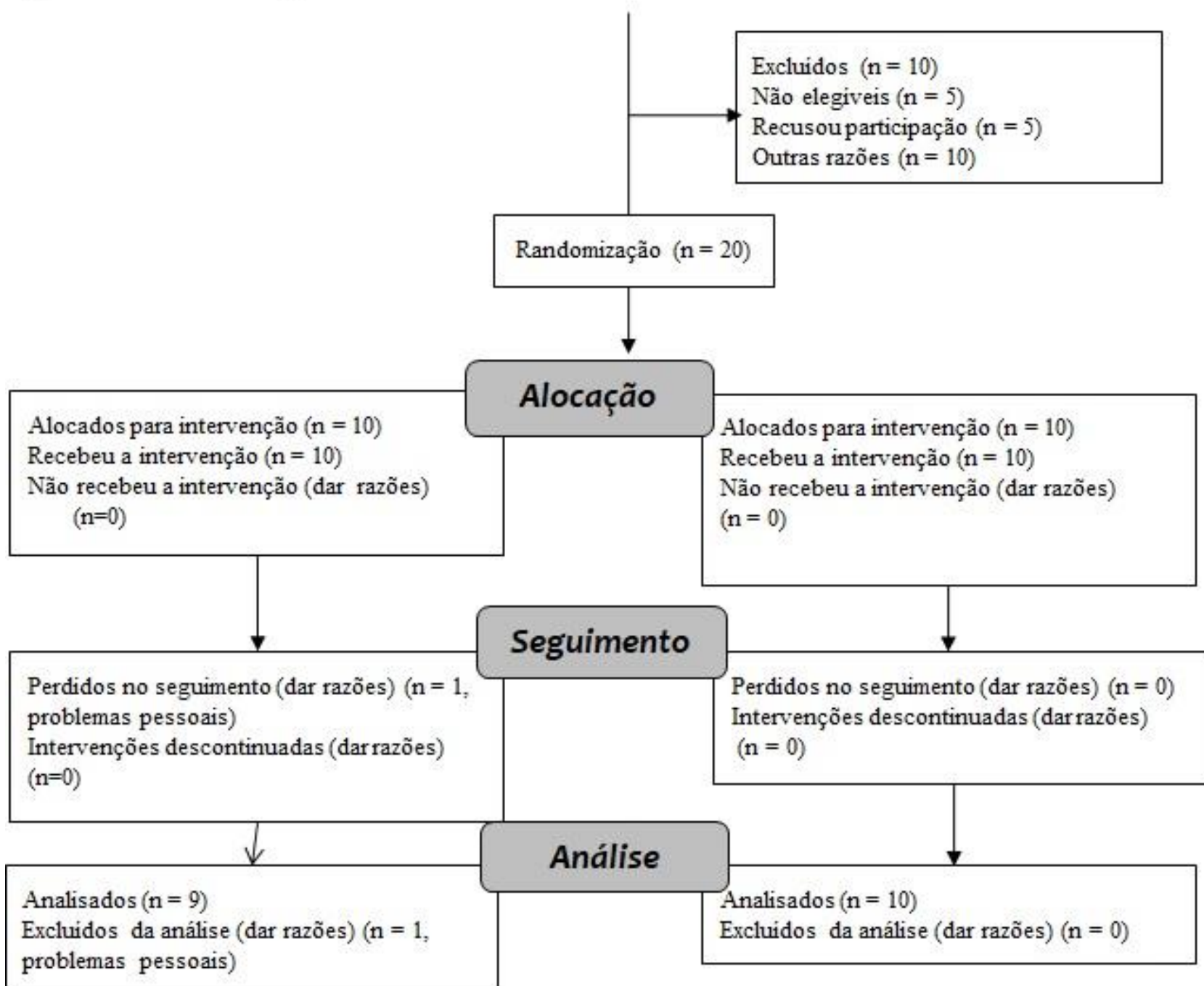

Figura 1 - Fluxograma.

Para coleta de dados, foi utilizada uma ficha de avaliação que constou de informações referentes aos dados de identificação, início do ganho de peso, fatores desencadeantes, tratamentos realizados, hábito alimentar, prática de exercícios físicos, história de tabagismo, presença de co-morbidades, antecedentes familiares de obesidade e de doenças cardiovasculares e o exame físico.

Os dados antropométricos foram devidamente aferidos: o peso, em balança digital Filizola; a altura, em estadiômetro Harpeden; as medidas da circunferência abdominal com 0 auxílio de fita métrica, avaliando as medidas da circunferência umbilical, na cintura com 5 e $10 \mathrm{~cm}$ acima e $5 \mathrm{~cm}$ abaixo da cicatriz umbilical (abdome) e a circunferência do quadril; e as dobras cutâneas, com adipômetro como recomendado pela Diretriz Brasileira de Diagnóstico e Tratamento da Síndrome Metabólica [10].

Foi realizado também o cálculo do IMC utilizando as medidas de peso e altura e a avaliação da composição corporal através das medidas das dobras cutâneas do tríceps braquial, suprailíaca e abdome para homens adultos. Para mulheres adultas, foi aferida a taxa de gordura corporal pelas medidas das dobras cutâneas do subescapular, supra ilíaca e coxa, realizadas com o adipômetro.

Todos os pacientes foram submetidos à orientação alimentar e, quando indicado, ao uso de drogas anorexígenas, condutas realizadas pelo endocrinologista como acontece em rotina no ambulatório. 
No tratamento fisioterápico dermatofuncional, os pacientes do grupo um (g1) foram submetidos a uma avaliação fisioterápica das patologias dermatofuncionais para obesidade, no início e ao final dos trinta atendimentos. A avaliação da gordura com uso do adipômetro foi realizada nas cinco medidas supracitadas para realização da composição da porcentagem de gordura antes e após o tratamento.

Nos atendimentos, os pacientes ( $\mathrm{g} 1$ ) foram submetidos a recursos dermatofuncionais como endermologia, no modo contínuo com pressão negativa de -150 a $-300 \mathrm{mmHg}$, conforme a tolerância do paciente, em um tempo de $15 \mathrm{~min}$; em seguida foi aplicado a corrente russa com frequência de $50 \mathrm{hz}$, com tempo de subida e descida da corrente (rise/decay) de $2 \mathrm{seg}$, tempo de contração e relaxamento, ambos de 15 segundos, por um tempo de 15 min e foi realizada a massagem modeladora, utilizando as técnicas de alisamento, torcedura, amassamento metacarpofalangiana e effleurage, por um período de $15 \mathrm{~min}$ na região abdominal, onde foi utilizado creme para massagem modeladora com princípio ativo de centella asiática, trietanolamina e cafeína. Após a aplicação dos recursos, os pacientes realizaram um programa ergométrico crescente em tempo de utilização com esteira eletrônica fit wake por $30 \mathrm{~min}$.

Os pacientes do grupo dois (g2) foram acompanhados no ambulatório de endocrinologia como grupo controle sem a realização da fisioterapia dermatofuncional.

Os dados obtidos foram analisados através de distribuição percentual das respostas fornecidas para cada questão, em relação à população utilizada no trabalho utilizando o programa estatístico prism $5.0(p<0,05)$ com two-way anova, com pós-teste de Bonferroni.

O protocolo para realização de pesquisa em seres humanos número 053/2011, aprovado pelo comitê de ética em pesquisa do centro universitário atestando que o projeto está dentro das normas que regulamentam a pesquisa em seres humanos, do Conselho Nacional de Saúde - Ministério da Saúde, resolução no. 466/2012.

Dos dez participantes que foram avaliados e tratados com técnicas fisioterápicas no grupo 1 (com intervenção), nove concluíram o tratamento e um desistiu por problemas pessoais. A análise dos dados obtidos nas avaliações sobre os dados antropométricos referentes ao IMC, às medidas da circunferência abdominal e pregas cutâneas foram expostas em forma de gráficos para comparar os resultados de antes e depois do tratamento fisioterápico.

Todos os pacientes do g1 iniciaram o tratamento com IMC acima do normal, com média de 30,5 \pm 4,23, 4 tinham sobrepeso e 6 tinham obesidade grau I, ao fim do tratamento todos permaneceram no mesmo grau de obesidade mais com valores menores, diminuindo para uma média de 29,9 .

A média dos valores da cicatriz umbilical foi de $101,4 \mathrm{~cm}$ no início da intervenção e ao final foi de $98,1 \mathrm{~cm}$ com diminuição de $3,3 \mathrm{~cm}$. Da cintura a $10 \mathrm{~cm}$ acima da cicatriz umbilical no início do tratamento, a média geral foi de $91,1 \mathrm{~cm}$ e ao final os pacientes tiveram uma média $88,1 \mathrm{~cm}$, ou seja, a perda foi de $3 \mathrm{~cm}$. Em relação à circunferência de $5 \mathrm{~cm}$ acima da cicatriz umbilical, a média foi de $93,8 \mathrm{~cm}$ no início e no final foi para $89,7 \mathrm{~cm}$ onde a perda foi de 4,1 $\mathrm{cm}$. No abdome, em $5 \mathrm{~cm}$ abaixo da cicatriz umbilical, a perda da circunferência do quadril foi de $2,5 \mathrm{~cm}$ com média inicial de $100,5 \mathrm{~cm}$ e final de $98,0 \mathrm{~cm}$. E a redução de medidas do quadril foi de 100,5 para $98,0 \mathrm{~cm}$ com perca de $2,5 \mathrm{~cm}$ (figura 2). Seja uma diminuição total de $15,4 \mathrm{~cm}$.

Para análise das medidas de índice cintura quadril (ICQ), e das cinco pregas cutâneas pode-se observar que a relação cintura/quadril estava acima do que é recomendado pela OMS, na qual o normal para os homens é até $1,0 \mathrm{~cm}$ e para as mulheres $0,8 \mathrm{~cm}$. A média geral antes do tratamento foi de $1,2 \mathrm{~cm}$ e após o tratamento obteve uma melhora para $0,9 \mathrm{~cm}$ indicando menor risco de doenças cardiovasculares.

As medidas realizadas para avaliar a gordura localizada foram realizadas utilizando 0 adipômetro. A mensuração da média de subescapular foi de $30,1 \mathrm{~mm}$ e ao final foi de $25,9 \mathrm{~mm}$ com perda geral de $4,2 \mathrm{~mm}$ com significância estatística $(p<0,05)$. Já no do tríceps braquial, foi identificada uma média inicial de $30,1 \mathrm{~mm}$ e média final de $27,9 \mathrm{~mm}$ com redução de 2,2 $\mathrm{mm}$, mas não obteve significância estatística. E as médias abdominal, supra ilíaca e coxa obtiveram melhora significativa $(p<0,01)$ com perda de $7,4 \mathrm{~mm}, 6,6 \mathrm{~mm}$ e $4 \mathrm{~mm}$ respectivamente (figura 3 ). 


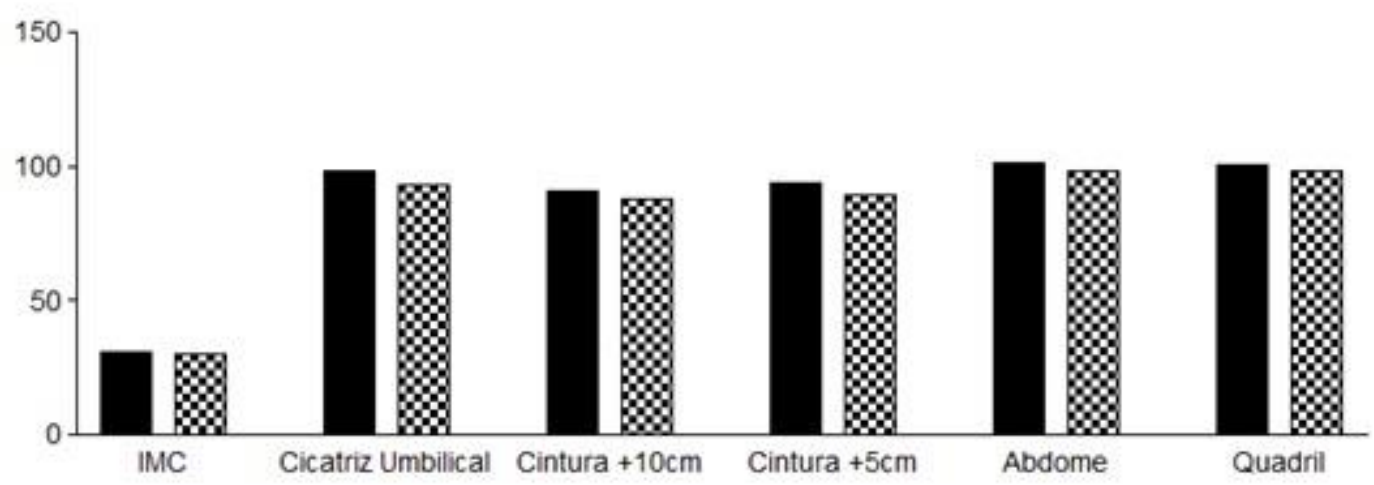

Antes

$\mathbf{\infty}$ Depois

IMC = índice de massa corporal.

Figura 2 - Avaliações dos dados antropométricos do grupo com intervenção fisioterápica antes e depois dos atendimentos. Média total de redução de medidas de 15,4 cm.

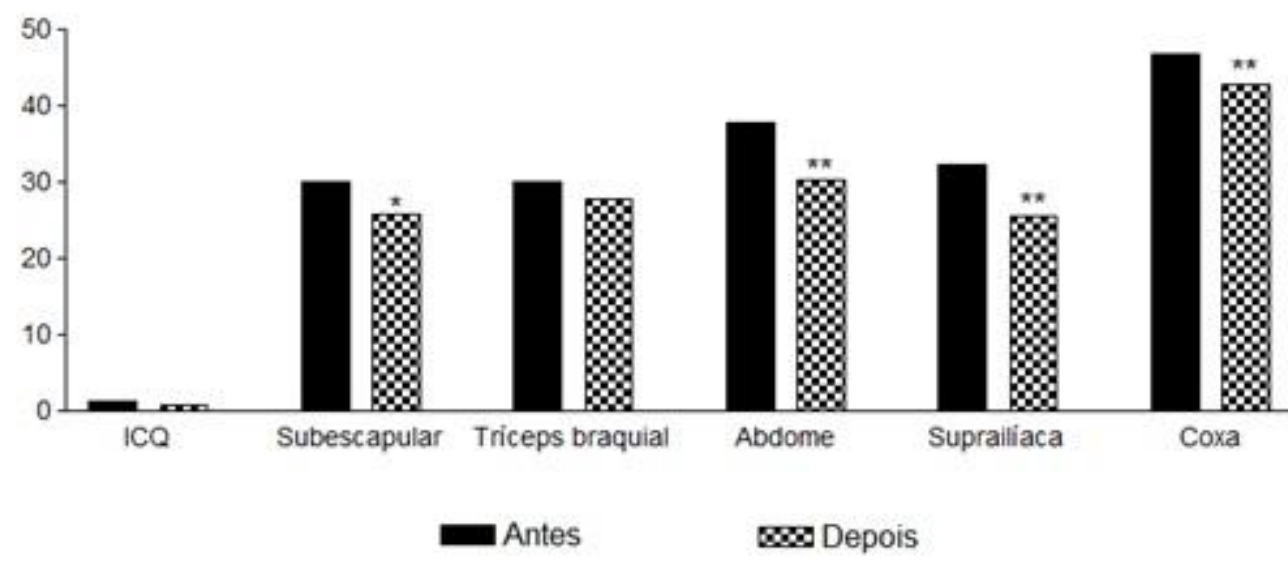

ICQ = índice de relação cintura/quadril; * $p<0,05 ;{ }^{* *} p<0,01$, two-way anova, com pós-teste de Bonferroni.

Figura 3 - Avaliações do ICQ e adipometria do grupo com intervenção fisioterápica antes e depois dos atendimentos. Média total de redução de medidas de 24,4 mm.

O g2 (sem intervenção) foi constituído por dez participantes, os quais foram avaliados duas vezes, sem nenhuma atuação fisioterápica, apenas acompanhamento. A média geral do IMC inicial desses pacientes foi 31,2 e na segunda avaliação foi de 31,5 obtendo um pequeno aumento.

A média da circunferência umbilical obteve um leve aumento de $1,7 \mathrm{~cm}$. Na medida da cintura de $10 \mathrm{~cm}$ acima da cicatriz umbilical houve, também, um leve aumento de $0,5 \mathrm{~cm}$, assim como $5 \mathrm{~cm}$ acima da cicatriz umbilical, no abdome e quadril, com leves aumentos de $1,1 \mathrm{~cm}$, $1,5 \mathrm{~cm}$ e $1,1 \mathrm{~cm}$, respectivamente (figura 4).

$\mathrm{Na}$ análise das medidas de ICQ, e das cinco pregas cutâneas do g2 (sem intervenção) pode-se observar que a relação também estava acima do que é recomendado pela OMS. A média geral antes do tratamento foi de $1,4 \mathrm{~cm}$ e após o tratamento obteve uma melhora para $1,0 \mathrm{~cm}$ indicando menor risco de doenças cardiovasculares, porém a redução não foi tanto quanto no grupo com intervenção.

A mensuração por adipômetro da região subescapular foi de média de $31,1 \mathrm{~mm}$ e ao final foi de $32,9 \mathrm{~mm}$ com ganho de $1,8 \mathrm{~mm}$. Já no do tríceps braquial, foi identificada uma média inicial de $29,1 \mathrm{~mm}$ e média final de $28,9 \mathrm{~mm}$ e na suprailíaca inicial de $32,2 \mathrm{~mm}$ e final de 33,6 com leve aumento de $0,2 \mathrm{~mm}$ e $1,4 \mathrm{~mm}$, respectivamente. $E$ as médias abdominal e coxa com reduções de $1,4 \mathrm{~mm}$ e $1 \mathrm{~mm}$. Dados sem significância estatística (figura 5). 


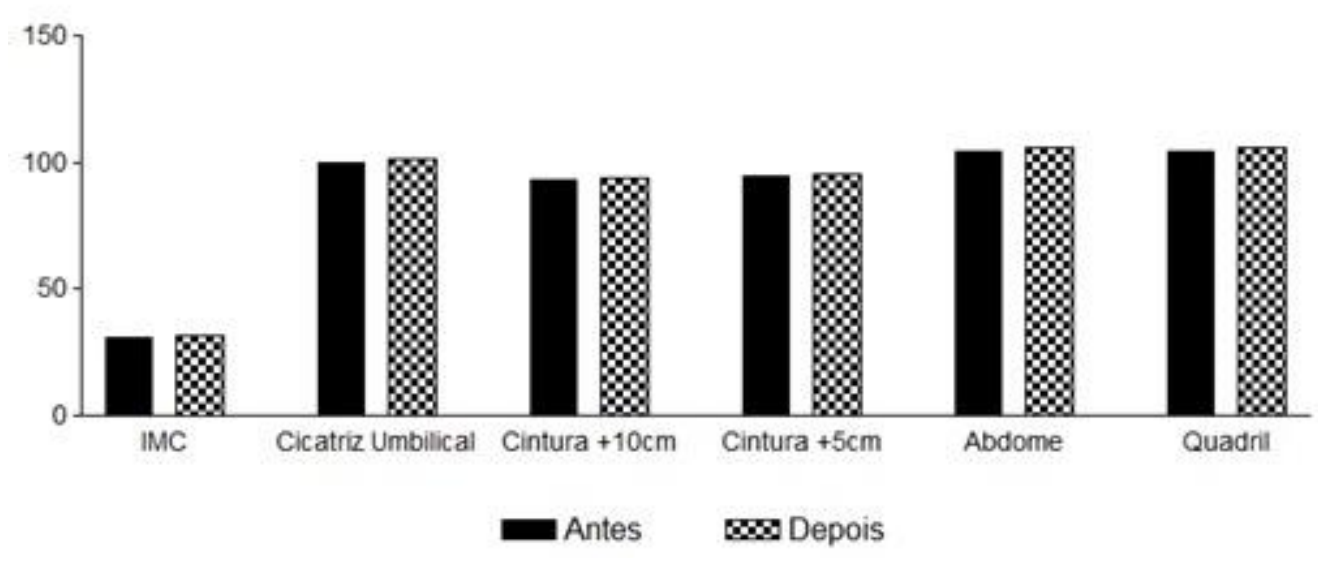

IMC = índice de massa corporal.

Figura 4 - Avaliações e reavaliações dos dados antropométricos do grupo controle (sem intervenção).

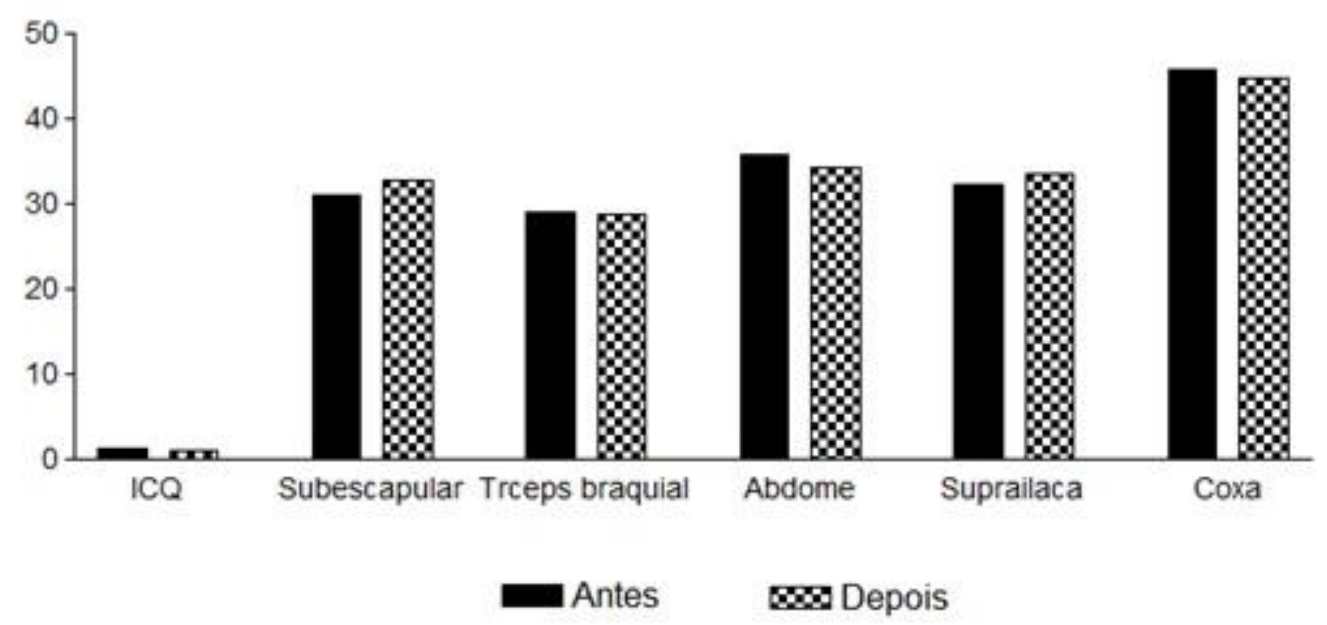

$I C Q=$ índice de relação cintura/quadril.

Figura 5 - Avaliações e reavaliações do ICQ e adipometria do grupo controle (sem intervenção).

\section{Discussão}

Desde 1975, a Organização Mundial Saúde passou a considerar a obesidade, em conjunto com a diabetes e a arteriosclerose, como problemas de saúde pública nos países desenvolvidos. Devido à superalimentação e de um estilo de vida inadequado a obesidade pode ser considerada como uma das enfermidades que pode estar presente em todas as faixas etárias $[11,12]$.

Como conduta de tratamento existem inúmeros recursos dermatofuncionais usados para gordura localizada, e um deles é a massagem modeladora que é uma técnica manual realizada através de movimentos fortes com maior pressão, produzindo um efeito mecânico, a fim de mobilizar e esvaziar as células adiposas, além de tonificar a musculatura local. Nessa técnica, são utilizados cremes que facilitam o manuseio do tecido adiposo, constituídos de princípios ativos que auxiliam na eliminação da gordura [13].

A massagem mecânica não invasiva do contorno corporal altera a distribuição da gordura subcutânea ou gordura localizada, por tracionar verticalmente os tecidos. Então, entende-se que as gorduras não são eliminadas, mas deslocadas, justificando o motivo da perda de peso dos pacientes do grupo que sofreu intervenção não ter sido significativa ao analisar o IMC [14].

Outros recursos indicados no tratamento da gordura localizada são os eletroterápicos, como a endermologia e a corrente russa que, juntos, proporcionam efeitos semelhantes ao da 
massagem modeladora. A endermologia consiste em uma técnica que envolve aparelhos específicos, baseados na sucção, realizando mobilização tecidual profunda, o que permite uma circulação sanguínea superficial satisfatória. Já a corrente russa, trata-se de uma estimulação elétrica neuromuscular de média frequência, que trabalha as fibras musculares vermelhas, responsáveis pela sustentação do corpo, melhorando a tonicidade muscular e o fluxo sanguíneo $[15,16]$.

A corrente russa é um recurso bastante utilizado para o fortalecimento muscular tanto em pacientes saudáveis como em pacientes em pós-operatório. Como efeitos fisiológicos produz melhores resultados quando associada a exercícios de contração muscular ativa promovendo respostas mais eficientes do que os exercícios isolados. No entanto, o presente estudo não teve como objetivo o fortalecimento muscular, mas foi observado que a mesma associada com o tratamento proposto, teve um valor significativo para a redução de medidas [16]. Porém, podemos observar que mesmo os locais que não receberam intervenção direta como a região subescapular, tríceps e coxa, obtiveram resultados significantes em relação aos locais que sofreram intervenção direta como o abdome. Os pacientes que participaram desse grupo de tratamento ficaram bastante satisfeitos com o resultado e muitos deles relataram que iriam continuar a re-educação alimentar associada aos exercícios físicos para manter a forma, o bem-estar e a saúde.

Usando as referidas técnicas da pesquisa combinadas, obtiveram-se resultados positivos, em geral, com diminuição em todas as circunferências e pregas cutâneas. Dados semelhantes aos de Migotto e Simões [17], que constataram em seu estudo resultados satisfatórios para o uso de massagem modeladora combinada a outros recursos.

O tecido adiposo é composto por adipócitos que aumentam em quantidade no primeiro ano de vida e na adolescência, período marcado pelo grande risco de se adquirir a obesidade, devido à multiplicação acelerada das células adiposas e pelas alterações emocionais, má alimentação e o sedentarismo [18]. O sedentarismo pode levar à flacidez muscular, que pode ocasionar dificuldade de circulação de retorno, com redução do fluxo sanguíneo o que pode levar a ativação da lipogênese. Em vista disso a gordura localizada não deve ser considerada apenas como uma desordem estética, e sim como uma alteração patológica [19].

Além dos recursos fisioterápicos, a participação em esportes e o aumento da atividade física são frequentemente recomendados no tratamento da obesidade, acompanhadas de orientação e educação alimentar. A ingestão energética inadequada também está associada à ingestão marginal de macro e micronutrientes, principalmente de carboidratos, piridoxina, cálcio, folato, zinco e magnésio, havendo consequências prejudiciais sobre o crescimento, tais como o aumento do risco de doenças e a diminuição da taxa metabólica [20]. Por isso, o grupo de tratamento, que realizou a atividade na esteira ergométrica, obteve melhor resultado que 0 grupo controle.

Os mecanismos pelos quais o excesso de peso e, sobretudo, a gordura visceral afetam o sistema cardiovascular incluem não somente o efeito indireto das comorbidades sobre a vasculatura (dislipidemia, hipertensão, apneia do sono, insulino-resistência), mas também o aumento da inflamação sistêmica e o alto turnover dos ácidos graxos livres com seus efeitos lipotóxicos. Também é citado o grau de comprometimento metabólico determinado e risco pela obesidade, que podem ser mensurados pela avaliação clínica ou laboratorial [20,21].

Existem múltiplas relações entre atividade física e obesidade. A atividade física diminui o risco de obesidade, atuando na regulação do balanço energético, influencia a distribuição do peso corporal, preservando ou mantendo a massa magra, além dos seus efeitos na redução do peso [22].

Fatores psicossociais e baixa aptidão física podem diminuir a motivação da pessoa obesa para a atividade física. A hipoatividade física cria um ciclo vicioso: inatividade - balanço calórico positivo - obesidade - diminuição da atividade física - maior inatividade. Desde pequena, a criança deve ser incentivada a uma vida mais ativa, pois o exercício realizado precocemente durante o período de crescimento previne a formação de novas células adiposas [23].

A criança em tratamento para a obesidade deve associar a ingestão calórica à prática de atividade física de baixa intensidade e larga duração, levando-se em consideração o aspecto recreacional. A perda de peso e os efeitos positivos cardiovasculares e respiratórios resultantes do condicionamento físico em obesos produzem diminuição do esforço fisiológico durante o exercício. No aspecto psicossocial, promove a melhora da autoimagem, autoconfiança, sociabilidade, além de menor percepção do esforço [24-26]. 
E o sedentarismo aumenta o risco de doenças e diminui a taxa metabólica. Por isso, também, o grupo de tratamento, que realizou a atividade na esteira ergométrica, obteve melhor resultado que o grupo controle [27-29]. Uma provável limitação deste estudo seria um adequado acompanhamento nutricional e de atividade física para ambos os grupos, pois o grupo de tratamento pode ter tido melhores resultados, pois, além do acompanhamento fisioterápico, foram estimulados a manter adequadamente a dieta alimentar, fato não observado no grupo não tratado que praticamente não houve alteração das medidas.

Conclusão

Conclui-se que o grupo que utilizou as referidas técnicas do estudo combinadas, tratamento endocrinológico associado às técnicas dermatofuncionais, obteve resultados mais satisfatórios, em relação à perda de medidas e a redução de risco dos marcadores, do que o grupo que utilizou somente o tratamento endocrinológico.

O estudo teve seu valor significativo demonstrando que os recursos da fisioterapia dermatofuncional não são utilizados somente para estética corporal, mas também para promover saúde, se for usado de forma consciente e responsável. Esse estudo pode ser visto como uma forma de incentivar as pessoas a se preocupar mais com a saúde e não somente com a estética corporal.

Referências

1. Radovanovic CAT, Santos LA, Carvalho MDB, Marcon SS. Hipertensão arterial e outros fatores de risco associados às doenças cardiovasculares em adultos. Rev Latinoam Enferm 2014;22(4):547-53.

2. Carvalho CA, Fonseca PCA, Barbosa JB, Machado SP, Santos AM, Silva AAM. Associação entre fatores de risco cardiovascular e indicadores antropométricos de obesidade em universitários de São Luís, Maranhão, Brasil. Ciênc Saúde Coletiva 2015;20(2):479-90.

3. Gus I, Ribeiro RA, Kato S, Bastos J, Medina C, Zazlavsky C, Portal VL et al. Variações na prevalência dos fatores de risco para doença arterial coronariana no Rio Grande do Sul: uma análise comparativa entre 2002-2014. Arq Bras Cardiol 2015;105(6):573-9.

4. Moro AK, Aldenucci BG. A atuação da fisioterapia dermato-funcional no pós-operatório de cirurgia bariátrica: uma revisão de literatura. Cinergis 2010;11(1):28-36.

5. Gomes EB, Moreira TMM, Pereira HCV, Sales IB, Freitas CHA, Rodrigues DP. Fatores de risco cardiovascular em adultos jovens de um município do nordeste brasileiro. Rev Bras Enferm 2012;65(4):594-600.

6. Mendonça RSC, Rodrigues GBO. As principais alterações dermatológicas em pacientes obesos. Abcd Arq Bras Cir Dig 2011;24(1):68-73.

7. Giuntoli ABB, Outa K, Monteiro PMA, Monomi PMA, Moraes SZ, Stulbach T. Comparação entre o índice de massa corpórea e porcentagem de gordura, e análise da relação cintura/quadril em desportistas de uma academia de São Paulo. Buenos Aires. Efdeportes.com 2012(171).

8. Martins MCC, Ricarte IF, Rocha CHL, Maia RB, Silva VB, Veras AB et al. Pressão arterial, excesso de peso e nível de atividade física em estudantes de universidade pública. Arq Bras Cardiol 2010;95(2):192-9.

9. Zaitune MPA, Barros MBA, César CLG, Carandina L, Goldbaum M. Fatores associados ao sedentarismo no lazer em idosos, Campinas, São Paulo, Brasil. Cad Saúde Pública 2007;23(6):1329-38.

10. Diretriz Brasileira de Diagnóstico e Tratamento da Síndrome Metabólica. Rev Soc Bras Hipert 2005;17(4).

11. Borges FS. Dermato-funcional: modalidades terapêuticas nas disfunções estéticas. 2.ed. São Paulo: Phorte; 2010.

12. Eckel RH, Barouch WW, Ershow AG. Report of the National Heart, Lung, and Blood Institute-National Institute of Diabetes and Digestive and Kidney Diseases working group on the pathophysiology of obesity-associated cardiovascular disease. Circulation 2002;105:2923-8.

13. Pereira PF, Serrano HMS, Carvalho GQ, Ribeiro SMR, Peluzio MCG, Franceschini SCC et al. Medidas de localização da gordura corporal: uma avaliação da colinearidade 
com massa corporal, adiposidade e estatura em adolescentes do sexo feminino. Rev Paul Pediatr 2015; 33(1):63-71

14. Pontes RB. Apostila do curso de massagem modeladora. Fortaleza: Pontes RB; 2008. p. 3-15.

15. Guirro ECO, Guirro RRJ. Fisioterapia dermato-funcional: fundamentos, recursos, patologias. 3. Ed. Rev. e ampl. São Paulo: Manole; 2006.

16. Kede MPV, Sabatovich O. Dermatologia estética. São Paulo: Atheneu; 2015.

17. Migotto JS, Simões NDP. Atuação fisioterapêutica dermatofuncional no pós-operatório de cirurgias plásticas. Rev G\&s 2013;4(1):1365-77.

18. Rosa QPP, Alves MK. Prevalência e fatores associados ao excesso de peso em mulheres adultas colaboradoras de uma unidade de alimentação e nutrição. Rbone 2017; 11(66):428-36.

19. Santos AA, Carvalho CC, Chaves ECL, Goyatá SLT. Qualidade de vida de pessoas com obesidade grau III: um desafio comportamental. Rev Bras Clin Med 2012;10(5):384-9.

20. Muniz LC, Schneider BC, Mohnsam ICS, Matijasevich A, Santos IS. Fatores de risco comportamentais acumulados para doenças cardiovasculares no sul do Brasil. Rev Saúde Pública 2012;46(3):534-42.

21. França IC, Akatsuka EW, Leal CP, Figueiredo MR, Oliveira LA, Andrade NS. Eficácia da técnica de massagem modeladora para redução de adiposidades e do fibroedemagelóide. Acis 2016;4(2):23-30.

22. Leite FO, Souza TV, Ribas TV, Francesconi MS, Bighetti AE. Avaliação de métodos estéticos de redução de gordura localizadas e mulheres sedentárias. Scientific Eletronic Archives 2017;10(2):73-7.

23. Araújo Mc, Bezerra IN, Barbosa FS, Junger WL, Yokoo EM, Pereira RA. Consumo de macronutrientes e ingestão inadequada de micronutrientes em adultos. Rev Saúde Pública 2013;47(suppl.1):177s-89s.

24. Faria APL. Atividade física, nutrição, farmacoterapia; a obesidade e seus tratamentos: uma revisão da literatura. Int J Nutrol 2017;10(3):91-8.

25. Fonseca-Junior SJ, Sá CGAB, Rodrigues PAF, Oliveira AJ, Fernandes-Filho J. Exercício físico e obesidade mórbida: uma revisão sistemática. Abcd Arq Bras Cir Dig 2013;26(suppl I):67-73.

26. Paes ST, Marins JCB, Andreazzi AE. Efeitos metabólicos do exercício físico na obesidade infantil: uma visão atual. Rev Paul Pediatr 2015;33(1):122-9.

27. Pernambuco AP, Carvalho NM, Santos AH. A eletroestimulação pode ser considerada uma ferramenta válida para desenvolver hipertrofia muscular? Fisioter Mov 2013;26(1):123-31.

28. Freitas MC, Ceschini FL, Ramallo BT. Resistência à insulina associada a obesidade:

29. Cruz e Silva AD, Castro AJO, Pereira APN, Souza AAR, Amorim PRA, Reis RC. Hábitos alimentares e sedentarismo em crianças e adolescentes com obesidade na admissão do programa de obesidade do Hospital Universitário Bettina Ferro de Souza. Rbone 2017;11(61):39-46. 